\title{
A Flexible Class of Models for Data Arising from a "thorough QT/QTc study"
}

\author{
Suraj P. Anand* and Sujit K. Ghosh \\ Department of Statistics, North Carolina State University, Raleigh, \\ North Carolina, USA
}

Institute of Statistics Mimeo Series 2610

\begin{abstract}
The standard methods for analyzing data arising from a 'thorough QT/QTc study' are based on multivariate normal models with common variance structure for both drug and placebo. Such modeling assumptions may be violated and when the sample sizes are small the statistical inference can be sensitive to such stringent assumptions. This article proposes a flexible class of parametric models to address the above mentioned limitations of the currently used models. A Bayesian methodology is used for data analysis and models are compared using the deviance information criteria (DIC). Superior performance of the proposed models over the current models is illustrated through a real data set obtained from a GlaxoSmithKline conducted 'thorough QT/QTc study'.
\end{abstract}

\section{Introduction}

QT prolongation is an important issue in clinical safely of already marketed and investigational new drugs. It is an undesirable property associated with some non-antiarrhythmic drugs due to their ability to delay cardiac repolarization, more generally known as QT prolongation. The QT interval is a segment of the surface electrocardiogram (ECG) and represents the duration of ventricular depolarization and subsequent repolarization, and is measured from the beginning of the QRS complex to the end of the T wave. QT values are correlated with heart rate and hence a corrected version (QTc) is used for data analysis (see International Conference of Harmonization (ICH) E14 guidelines, available at www.fda.gov/cder/guidance/). The ICH

\footnotetext{
${ }^{*}$ Correspondence: Suraj Anand, Department of Statistics, North Carolina State University, Raleigh, NC 27695-8203, USA; E-mail: spanand@ncsu.edu
} 
E14 guidelines recommend conducting a 'thorough QT/QTc study' to determine whether the drug has a threshold pharmacological effect on cardiac repolarization, as detected by QTc prolongation. The study is typically carried out in healthy volunteers and is used to determine whether or not the effect of a drug on the QTc interval in target patient populations should be studied intensively during later stages of drug development.

Measurements taken in a QT study are naturally in time order, with multiple QTc measurements taken on each subject at a number of time-points, hence the comparison of drug with placebo involves assessing the time-matched differences at each of those time-points. The standard approach to analyzing QT data to assess a drug for a potential QT prolongation effect is driven by an intersection-union testing approach. This is performed by constructing a 90\% two-sided (or a 95\% one-sided) confidence interval (CI), for the time-matched mean difference in baseline corrected QTc between drug and placebo at each time-point, and concluding non-inferiority if the upper limits for all these CIs are less than 10 ms. A positive control known to have a QT prolonging effect is also included to assess the sensitivity of the study in detecting the QT effects. Under the standard normality assumptions, the intersection-union test based standard approach is known to be conservative and biased (Patterson and Jones, 2005).

According to the The ICH E14 guidelines, a negative 'thorough QT/QTc Study' is one in which the upper bound of the $95 \%$ one-sided confidence interval for the largest time-matched mean effect of the drug on the QTc interval excludes $10 \mathrm{~ms}$. When the largest time-matched mean difference exceeds the threshold, the study is termed positive. Hence, instead of constructing confidence intervals at each of the time-points , one could base the non-inferiority inference on an interval estimate for the largest time-matched mean difference in QTc between drug and placebo. Constructing such an interval estimate for the maximum difference, which is a non-smooth function, however is a non-trivial task.

Few notable attempts to address this problem include newer approaches proposed by Eaton et al. (2006), Boos et al. (2007), and Anand and Ghosh (2008). The method proposed by Eaton et al. (2006) involves constructing asymptotic confidence interval for the parameter of maximum difference by approximating it with a smooth function and then using the 'delta method' to obtain an approximate confidence interval. In another study, Boos et al. (2007) proposed new bounds for the maximum difference, which are based on analytical and bootstrap methods. The first upper bound that they propose can be 
obtained analytically and uses an expression for the approximate moments of the maximum of random variables. Their second upper bound is based on a parametric bootstrap, bias-corrected percentile method, whereas their third upper bound is based on a non-parametric bootstrap, bias-corrected percentile method. Recently, Anand and Ghosh (2008) proposed a Bayesian approach to solving this problem. Their approach involves constructing an interval estimate for the maximum difference based on the derived samples from the posterior density of the maximum difference. They use normal conjugate priors for the mean vectors and inverse wishart prior for the common covariance matrix to derive the closed form posterior density for the population mean vectors and covariance matrix, and employ a Monte Carlo sampling technique to sample from the posterior density of the maximum difference. Their proposed approach is based on finite sample and does not make use of any asymptotic approximations.

All the above methods are based on multivariate normal models for the QT data with common covariance structure for both drug and placebo. Such modeling assumptions may not be reasonable in all situations and may need to be relaxed to reflect observed data. These assumptions may be violated and when the sample sizes are small the statistical inference about the maximum time-matched mean difference parameter can be sensitive to such stringent assumptions. In this Chapter, we propose a flexible class of parametric models to address the above mentioned limitations of the currently used models. A Bayesian methodology is used for data analysis and we compare the models using the deviance information criteria (DIC). We illustrate the superior performance of the proposed models over the current models through a real data set obtained from a GSK conducted Thorough QT study. We lay out a flexible class of multivariate models suitable for the QTc data in Section 2. In Section 3, we provide details for the model comparisons and analysis methods. In Section 4 we apply the proposed method to a real data set on QTc obtained from a thorough QT study run at GlaxoSmithKline (GSK). We compare different models using the DIC criterion and provide analysis results for the preferred model. Finally, We present the concluding remarks and a discussion in Section 5. 


\section{A Flexible Class of Models for QTc Data}

We develop a general statistical model for the parallel study design for QTc data. Let $n_{1}$ and $n_{2}$ denote the number of subjects receiving drug and placebo respectively, with baseline corrected QTc values calculated at $p$ time points. We denote the vector of $p$ measurements taken on the subjects on drug by $\boldsymbol{x}_{i}, i=1, \ldots n_{1}$, and those on control by $\boldsymbol{y}_{j}, j=1, \ldots n_{2}$, respectively. The commonly used models for QTc data are all based on the assumptions of normality and common covariance structure for drug and placebo but we allow a much more flexible class of multivariate $t$ models in all its generality. We assume that the measurements are distributed identically and independently (iid), arising from two independent $p$-variate $t$ distributions as follows:

$$
\begin{aligned}
& \boldsymbol{x}_{i} \stackrel{i i d}{\sim} \boldsymbol{t}_{\nu_{1}}\left(\boldsymbol{\mu}_{1}, \boldsymbol{\Sigma}_{1}\right), \quad i=1, \ldots, n_{1}, \text { and } \\
& \boldsymbol{y}_{j} \stackrel{i i d}{\sim} \boldsymbol{t}_{\nu_{2}}\left(\boldsymbol{\mu}_{2}, \boldsymbol{\Sigma}_{2}\right), \quad j=1, \ldots, n_{2},
\end{aligned}
$$

where $\boldsymbol{\mu}_{1}$ and $\boldsymbol{\mu}_{2}$ are the population means for the drug and placebo groups, respectively, and the dispersion matrices $\boldsymbol{\Sigma}_{1}$ and $\boldsymbol{\Sigma}_{2}$ are assumed to be unknown and unstructured. We denote by $\boldsymbol{\delta}=\boldsymbol{\mu}_{1}-\boldsymbol{\mu}_{2}$, the vector of time-matched mean differences in mean QTc between drug and placebo. $\nu_{1}>2$ and $\nu_{2}>2$ are the degrees of freedom for the respective $t$ distributions. The parameter of interest is the maximum time-matched mean difference between the two groups and we denote it by $\theta$,

$$
\theta=\max _{1 \leq k \leq p} \delta_{k} \equiv \max _{1 \leq k \leq p}\left(\mu_{1 k}-\mu_{2 k}\right)
$$

It is well known that as the degrees of freedom get large $\nu_{k} \rightarrow \infty$ for $k=1,2$, the multivariate $t$ distribution approaches to a multivariate normal distribution and hence this flexible class of models includes the commonly used multivariate models for the QTc data.

The primary objective of a QT study is to obtain an interval estimate for $\theta$. It is easy to see that $\theta$ is a non-smooth function of $\boldsymbol{\mu}_{1}$ and $\boldsymbol{\mu}_{2}$ and hence we can not apply the delta-method (Casella and Berger, 2002, p.245) based on the moment estimates $\hat{\boldsymbol{\mu}}_{1}=\overline{\boldsymbol{x}}$ and $\hat{\boldsymbol{\mu}}_{2}=\overline{\boldsymbol{y}}$, where $\overline{\boldsymbol{x}}=\sum_{i} \boldsymbol{x}_{i} / n_{1}$ and $\overline{\boldsymbol{y}}=\sum_{j} \boldsymbol{y}_{j} / n_{2}$ denote the sample means. Also, unlike the case with normal likelihood, there does not exist a class of conjugate 
priors for this general class of models. So, depending on the prior specifications on $\boldsymbol{\mu}_{1}, \boldsymbol{\mu}_{2}, \boldsymbol{\Sigma}_{1}$ and $\boldsymbol{\Sigma}_{2}$, the respective posterior densities are analytically intractable but we can still sample from these posterior distributions using Markov Chain Monte Carlo (MCMC) sampling techniques. It should be noted that even with the simpler Multivariate normal model, for which a class of conjugate priors exists for $\left(\boldsymbol{\mu}_{1}, \boldsymbol{\mu}_{2}, \boldsymbol{\Sigma}\right)$, where $\boldsymbol{\Sigma}$ is the common covariance matrix $\left(\boldsymbol{\Sigma}_{1}=\boldsymbol{\Sigma}_{2}=\boldsymbol{\Sigma}\right)$, it is still almost impossible to obtain the posterior distribution of $\theta$ analytically and one has to employ a Monte Carlo sampling method to generate samples from this posterior distribution (Anand and Ghosh, 2008 ).

In order to obtain the posterior distribution of $\theta$ we first specify a class of priors for $\boldsymbol{\mu}_{1}, \boldsymbol{\mu}_{2}, \boldsymbol{\Sigma}_{1}$ and $\boldsymbol{\Sigma}_{2}$. A suitable class of priors for this model can be given as follows:

$$
\begin{aligned}
\boldsymbol{\mu}_{1} & \sim \mathcal{N}_{p}\left(\boldsymbol{\mu}_{10}, \boldsymbol{\Sigma}_{10}\right), \\
\boldsymbol{\mu}_{2} & \sim \mathcal{N}_{p}\left(\boldsymbol{\mu}_{20}, \boldsymbol{\Sigma}_{20}\right), \\
\boldsymbol{\Sigma}_{1}^{-1} & \sim \mathcal{W}_{p}\left(k, \boldsymbol{R}_{10}\right) \text { and } \\
\boldsymbol{\Sigma}_{2}^{-1} & \sim \mathcal{W}_{p}\left(k, \boldsymbol{R}_{20}\right),
\end{aligned}
$$

where $\mathcal{W}_{p}$ denotes a $p$-dimensional Wishart distribution with degrees of freedom $k>p-1$ and positive definite scale matrix $\boldsymbol{R}_{j 0}, j=1,2$ (Anderson, 1984, p.245).

With this class of priors, the posterior distribution of $\left(\boldsymbol{\mu}_{1}, \boldsymbol{\mu}_{2}, \boldsymbol{\Sigma}_{1}, \boldsymbol{\Sigma}_{2}\right)$, and hence $\theta$, is not analytically tractable. So, one has to employ MCMC sampling techniques to generate samples from the posterior distribution of $\theta$ and use them to construct an interval estimate for $\theta$.

\section{Model Comparison and Analysis Methodology}

We start with the commonly used mutivariate normal model with a common covariance structure for drug and placebo, which is a special case of the general multivariate $t$ model when $\nu_{k} \rightarrow \infty$ for $k=1,2$ and $\boldsymbol{\Sigma}_{1}=\boldsymbol{\Sigma}_{2}=\boldsymbol{\Sigma}$. To ascertain the validity of the assumption of same covariance structure for drug and 
placebo, we perform Box's M test (Box, 1949) on the estimated covariance matrices. To do this, we calculate the $U$ statistic given by the formula $U=-2\left(1-c_{1}\right) \ln M$, where

$$
\begin{aligned}
& \ln M=\frac{1}{2} \sum_{1}^{k} r_{i} \ln \left|S_{i}\right|-\frac{1}{2}\left(\sum_{1}^{k} r_{i}\right) \ln \left|S_{p l}\right|, \\
& c_{1}=\left(\sum_{1}^{k} \frac{1}{r_{i}}-\frac{1}{\sum_{1}^{k} r_{i}}\right)\left(\frac{2 p^{2}+3 p-1}{6(p+1)(k-1)}\right),
\end{aligned}
$$

and $S_{p l}=\frac{\sum_{1}^{k} r_{i} S_{i}}{\sum_{1}^{k} r_{i}}$, where $S_{i}$ is the estimated covariance matrix for $i$ th arm, $r_{i}=n_{i}-1$ is the degrees of freedom associated with the $i t h$ arm, $p$ is the dimension of the data vector, and $k=2$ is the number of arms. Under the normality assumption for the data, $U$ follows a Chi square distribution with $\frac{1}{2}(k-1) p(p+1)$ degrees of freedom (Box, 1949).

If the Box's M test yields a significant result, we abandon the common covariance assumption, otherwise we employ a model selection technique to select the better model. An objective criterion to compare the models could be based on the ability of the model to best predict a replicate dataset which has the same structure as that currently observed one, and hence we use the Deviance Information Criterion (DIC) proposed by Spiegelhalter et. al., (2002), for model comparisons. We choose a model with the smallest DIC among a collection of finitely many models. There is no set significance rule for preferring one model over another using DIC but a difference of more than 10 might rule out the model with the higher DIC, differences between 5 and 10 are substantial, and differences less than 5 need to be used with caution. These suggested guidelines are available at www.mrc-bsu.cam.ac.uk/bugs/winbugs/dicpage.shtml.

For the normal models, we employ the above mentioned scheme to decide whether the assumption of a common covariance structure for drug and placebo is a reasonable one, and then use DIC to further compare the models. We also use DIC to compare the multivariate normal and $t$ models. For multivariate $t$ models, as we indicated above, putting priors on the degrees of freedom $\nu_{k}$ for $k=1,2$ makes sampling from the posterior distributions too difficult, so we assume that $\nu_{1}=\nu_{2}=\nu$ is fixed and treat it as a tuning parameter. To select the appropriate $\nu$, we calculate the DIC for a range of $\nu$ s and choose the one that minimizes the DIC for the model. We also use the DIC to see how a change in prior information affects the results. 
In the absence of a closed form posterior density for $\theta$, we used the MCMC software Winbugs (WinBUGS, version 1.4.1; available at www.mrc-bsu.cam.ac.uk/bugs/winbugs/) for generating samples from the posterior distribution of $\boldsymbol{\mu}_{1}, \boldsymbol{\mu}_{2}, \boldsymbol{\Sigma}_{1}$ and $\boldsymbol{\Sigma}_{2}$, and hence $\theta$. Upto three chains with different initial values were used to diagnose convergence. Programming language R (available at www.r-project.org/) was used to load data into Winbugs, perform data manipulations, call and execute Winbugs from $\mathrm{R}$ using the R package R2WinBUGS (also available at www.r-project.org/). DIC for each model was computed using WinBUGS. Let $D(\boldsymbol{\theta})=-2 \log f(\boldsymbol{x}, \boldsymbol{y} \mid \boldsymbol{\theta})$ denote the deviance for the model where $\boldsymbol{\theta}=\left(\boldsymbol{\mu}_{1}, \boldsymbol{\mu}_{2}, \boldsymbol{\Sigma}_{1}, \boldsymbol{\Sigma}_{2}\right)$, where $f(\boldsymbol{x}, \boldsymbol{y} \mid \boldsymbol{\theta})$ denotes the joint density of $(\boldsymbol{x}, \boldsymbol{y})$ given $\boldsymbol{\theta}$. If we denote by $\hat{D}$ the point estimate of the deviance obtained by substituting in the posterior mean $\overline{\boldsymbol{\theta}}=E[\theta \mid \boldsymbol{x}, \boldsymbol{y}]$,

$$
\hat{D}=-2 \log f(\boldsymbol{x}, \boldsymbol{y} \mid \overline{\boldsymbol{\theta}})
$$

and by $\bar{D}$ the posterior mean of the deviance $\bar{D}=E[D(\boldsymbol{\theta}) \mid \boldsymbol{x}, \boldsymbol{y}]$, then the effective number of parameters $p_{D}$ is given by

$$
p_{D}=\bar{D}-\hat{D}
$$

DIC is then defined as

$$
D I C=\bar{D}+p_{D}=\hat{D}+2 p_{D}
$$

$\hat{D}$ gives a measure of goodness of fit of the model whereas $p_{D}$ evaluates a measure of model complexity. DIC combines these two characteristics together to give a model assessing criterion in a way such that smaller values indicate a better predictive ability of a model.

The samples generated from the posterior distribution of $\theta$ were used to calculate the $95 \%$ quantile as an interval estimate for the maximum time-matched mean difference $\theta$. Samples generated from the posterior distributions of $\boldsymbol{\Sigma}_{1}$ and $\boldsymbol{\Sigma}_{2}$ were used to obtain the posterior mean covariance matrices for drug and placebo, and these estimates were used to check the common covariance assumption using Box's M test. 


\section{Real Data Example, Analysis and Results}

Change from baseline is the primary end-point of interest in a QT study, where baseline is defined according to the study design, the dosing pattern and other study specific considerations.

Table 1: Data schematics for a typical QT study.

\begin{tabular}{r|r|r|r|r|r|r}
\hline \hline Subject & Time (Hrs) & 1 & 2 & 3 & $\ldots \ldots$ & 24 \\
\hline 1 & Baseline QTc & $\mathrm{X} 1$ & $\mathrm{X} 2$ & $\mathrm{X} 3$ & $\ldots \ldots$. & $\mathrm{X} 24$ \\
1 & Post-Dose (Drug) & $\mathrm{Y} 1$ & $\mathrm{Y} 2$ & $\mathrm{Y} 3$ & $\ldots \ldots$. & $\mathrm{Y} 24$ \\
1 & Change from baseline (Drug) & $\mathrm{Y} 1-\mathrm{X} 1$ & $\mathrm{Y} 2-\mathrm{Y} 1$ & $\mathrm{Y} 3-\mathrm{X} 3$ & $\ldots \ldots$. & $\mathrm{Y} 24-\mathrm{X} 24$ \\
\hline 2 & Baseline QTc & $\mathrm{X} 1$ & $\mathrm{X} 2$ & $\mathrm{X} 3$ & $\ldots \ldots$. & $\mathrm{X} 24$ \\
2 & Post-Dose (Placebo) & $\mathrm{Y} 1$ & $\mathrm{Y} 2$ & $\mathrm{Y} 3$ & $\ldots \ldots$ & $\mathrm{Y} 24$ \\
2 & Change from baseline (Placebo) & $\mathrm{Y} 1-\mathrm{X} 1$ & $\mathrm{Y} 2-\mathrm{Y} 1$ & $\mathrm{Y} 3-\mathrm{X} 3$ & $\ldots \ldots$. & $\mathrm{Y} 24-\mathrm{X} 24$ \\
\hline \hline
\end{tabular}

Table 1 presents the data schematics of a typical QT study. Multiple (usually triplicate) measurements are taken at baseline to provide a mean baseline measurement by averaging across the replicates. Usually, a positive control that is known to have an increasing effect on the QTc interval is also included to assess the sensitivity of the study in detecting the QT effects. If the positive control demonstrates an effect as compared to placebo, then the assay is considered reasonably sensitive to detection of QTc prolongation.

We use the data previously analyzed by Anand and Ghosh, (2008) that were obtained from a GSK conducted thorough QT study with placebo, a positive control (Moxifloxacin), and multiple drug concentration arms for the study drug, with QTc (Bazett) measurements taken at baseline (mean of triplicates) and multiple time-points $(p=10)$. Although the actual study design was crossover, only data from a single period of the entire dataset were used and only data on placebo and the positive control arm (called drug henceforth) were included to mimic a parallel study design, with 36 subjects in each arm, for the purpose of illustration. The end-point of interest was change from baseline QTc values and the parameter of interest was maximum time-matched mean difference between drug and placebo for baseline corrected QTc. The primary aim of this research is to compare several models and obtain an interval estimate of $\theta$ based on the best fitting model. 


\subsection{Robustness With Respect to Sampling Distribution}

We focussed our attention to the models that are indicated above in Section 3. The first model (M1) is the simplest model that assumes that the data for drug and placebo arise from two multivariate normal populations with different means but the same covariance structure. This corresponds to the special case of model (1) where $\boldsymbol{\Sigma}_{1}=\boldsymbol{\Sigma}_{2}$ and $\nu_{1}=\nu_{2}=\infty$. The second model (M2) again assumes that the data come from multivariate normal populations, but with different covariance structures for drug and placebo. The third model (M3) assumes that the data come from multivariate $t$ distributions with same and fixed degrees of freedom (df), $\nu_{1}=\nu_{2}=11$, with same covariance structures for drug and placebo, whereas, the fourth model (M4) is a variation of the third model with different covariance structures for drug and placebo.

Table 2: Posterior summary statistics for $\theta$ using different models for the GSK data.

\begin{tabular}{l|r|r|r|r|r}
\hline \hline$t$ Model & mean & sd & $95 \%$ ile & DIC & $p_{D}$ \\
\hline M1: $\boldsymbol{\Sigma}_{1}=\boldsymbol{\Sigma}_{2} ; \nu_{1}=\nu_{2}=\infty$ & 15.4 & 2.2 & 19.0 & 5268.5 & 69.5 \\
M2: $\boldsymbol{\Sigma}_{1} \neq \boldsymbol{\Sigma}_{2} ; \nu_{1}=\nu_{2}=\infty$ & 15.5 & 2.3 & 19.2 & 5311.6 & 110.1 \\
M3: $\boldsymbol{\Sigma}_{1}=\boldsymbol{\Sigma}_{2} ; \nu_{1}=\nu_{2}=\nu^{*}$ & 14.9 & 2.2 & 18.5 & 4773.0 & 66.5 \\
M4: $\boldsymbol{\Sigma}_{1} \neq \boldsymbol{\Sigma}_{2} ; \nu_{1}=\nu_{2}=\nu$ & 14.7 & 2.2 & 18.3 & 4784.9 & 108.2 \\
\hline${ }^{*}=11$ for the results provided in this table
\end{tabular}

We used Winbugs to generate a total of 15000 samples from the posterior distribution of $\boldsymbol{\mu}_{1}, \boldsymbol{\mu}_{2}$, $\boldsymbol{\Sigma}_{1}$ and $\boldsymbol{\Sigma}_{2}$, and hence $\theta$ followed by a burnin of 15000 samples based on three parallel chains. We used a class of normal priors for $\boldsymbol{\mu}_{1}, \boldsymbol{\mu}_{2}$ with the hyperparameters $\boldsymbol{\mu}_{j 0}=0$ for $j=1,2$ and $\boldsymbol{\Sigma}_{j 0}=10^{2} \boldsymbol{I}_{p}, j=1,2$, and an inverted wishart prior for $\boldsymbol{\Sigma}_{j}$ with the hyperparameters $k=p+2$ and $\boldsymbol{R}_{j 0}=\boldsymbol{I}_{p}$ for $j=1,2$. Data and the intial values were loaded into Winbugs using $\mathrm{R}$, and Winbugs execution was performed using the $\mathrm{R}$ package R2WinBUGS. DIC and $p_{D}$ for each model were computed using Winbugs. The samples generated from the posterior distribution of $\theta$ for the above mentioned models were used to construct an upper $95 \%$ interval estimate (95\% quantile) for the maximum time-matched mean difference $\theta$. Samples generated from the posterior distributions of $\boldsymbol{\Sigma}_{1}$ and $\boldsymbol{\Sigma}_{2}$ were used to obtain the posterior mean covariance matrices for drug and placebo, and these estimates were used to check the common covariance assumption using Box's M test. We present these results in Table 2. We also present the posterior densities of $\theta$ under the four models in Figure 1. 


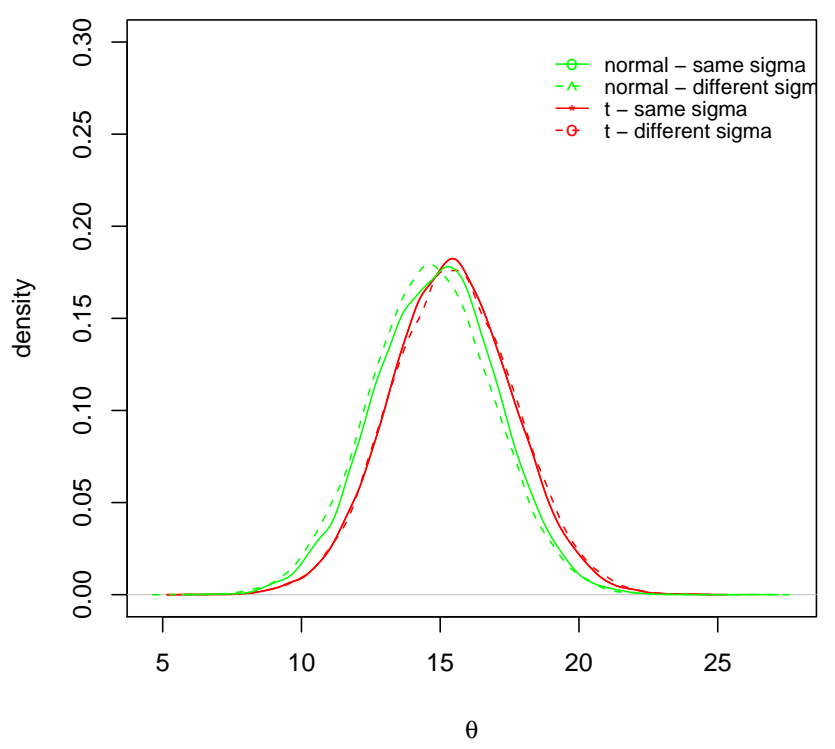

Figure 1: Posterior Density Plots for $\theta$ for different models

Looking at the summary statistics presented in Table 2 and Figure 1, it appears that even a change in distributional assumptions for the data does not have a significant impact on the inference for $\theta$. The means, standard deviations, and the $95 \%$ quantiles for $\theta$ for the multivariate $t$ models (models M3 and M4) are slightly lower than that for the multivariate normal models (models M1 and M2), but the difference is not substantial. Thus, the inference for $\theta$ appears to be reasonably robust against changes to distributional assumptions for the data.

It is easy to see that the DIC is much larger for the multivariate normal models (M1 and M2) as compared to the multivariate $t$ models (M3 and M4), which indicates that, based on the minimum expected predictive error criterion as indicated by a lower DIC, the multivariate $t$ models are expected to perform better than the multivariate normal models for the given data. Between the two multivariate $t$ models, the one with same covariance structure for both drug and placebo outperforms the $t$ model with different covariance structures. To ascertain the validity of the assumption of same covariance structure for drug and placebo, we performed Box's M test on the estimated covariance matrices as explained in Section 3. The values of $U$ for the multivariate normal and $t$ models with different covariance structures came out to be 
50.18 and 45.08 respectively, which when compared with the critical value $\chi_{55,0.05}^{2}=73.31$ suggest that the assumption of a common covariance structure seems reasonable. These results indicate that a multivariate $t$ model with fixed degrees of freedom and same covariance structure seems to be the most appropriate choice for the data. So, we proceeded to further investigate model M3 in greater details.

In models M3 and M4, we fixed degrees of freedom $\nu=11$ as an initial guess to check the performance of the $t$ models. To make a DIC-based selection of the degrees of freedom (df) for model M3, we calculated the DIC for a range of $\nu$ s and chose the one that minimizes the DIC for model M3. Figure 2 (a) presents a plot of DIC vs. $\nu$ for this model. It can be seen that the DIC decreases with df until $\nu=6$, and then increases consistently with df. We also present a plot for the $95 \%$ quantile for $\theta$ vs. $\nu$ in Figure 2 (b). The plot suggests that the $95 \%$ quantile for $\theta$ is not affected much by a change in $\nu$. This suggests that $\nu=6$ is the most appropriate choice for model M3 according to the DIC criterion. Table 3 presents the results for the multivariate $t$ model with $\nu=6$ and same covariance structure for drug and placebo.

(a)

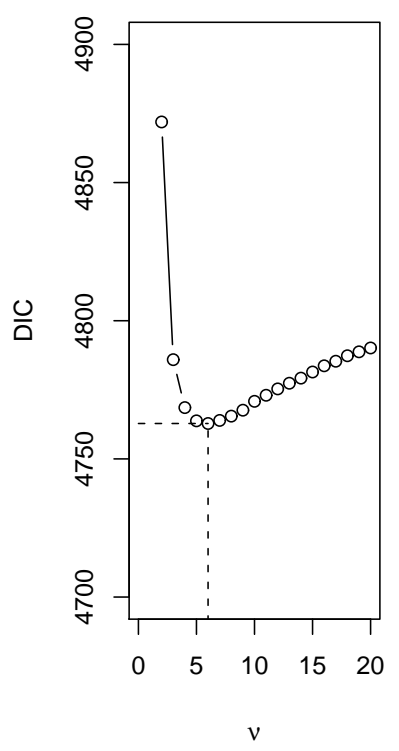

(b)

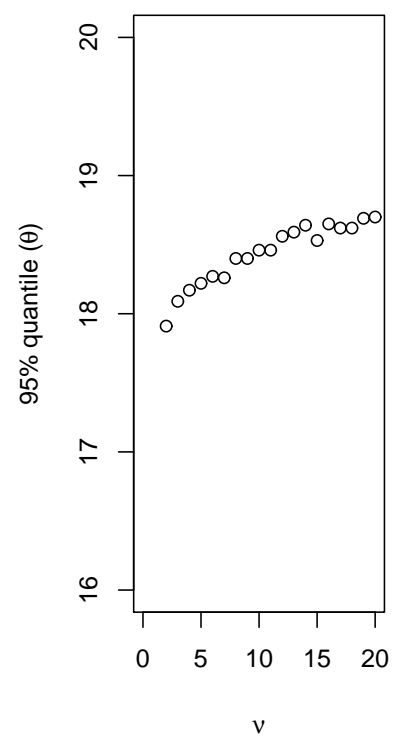

Figure 2: For model M3 with $\nu$ degrees of freedom, panel (a) presents DIC vs. $\nu$ and panel (b) presents the $95 \%$ posterior quantile of $\theta$ vs. $\nu$ 
Table 3: Posterior summary statistics for $\theta$ using model M3 with $\nu=6$.

\begin{tabular}{l|r|r|r|r|r}
\hline \hline Model & mean & sd & $95 \%$ ile & DIC & $p_{D}$ \\
\hline M3 ${ }^{*} \boldsymbol{\Sigma}_{1}=\boldsymbol{\Sigma}_{2} ; \nu_{1}=\nu_{2}=6$ & 14.7 & 2.2 & 18.3 & 4762.8 & 67.9 \\
\hline \hline
\end{tabular}

\subsection{Robustness With Respect to Prior Specifications}

After ascertaining the robustness of our methodology for distributional assumptions for the data, it was also of interest to see how changes to prior information affect the results. To do this, we first investigated the sensitivity of our methodology to the hyperparameter $k$ that appears to have a significant effect on the DIC of the model. We calculated the DIC for a range of values of $k$ for model M3* (with $\nu=6$ ) as selected above. Figure 3 (a) presents a plot of DIC vs. $k$ for this model. It can be seen that the DIC slowly decreases with $k$ until $k=17$ and then shoots up rapidly. This suggests that $k=17$ is the most appropriate choice for model M3 according to the DIC criterion. Even though the DIC increases rapidly with $k$, the inference for $\theta$ does not change sharply. For example, the $95 \%$ quantile for $\theta$ for $k=12$ is 18.3 as compared to 18.0 for $k=40$, and the two numbers are not too different for regulatory decisions. This shows that even though the predictive ability of the model decreases with an increase in $k$, the inference for $\theta$ remains robust to this change. Table 4 presents the results for $k=17$ and few extreme values of $k$. We also present a plot for the 95\% quantile for $\theta$ vs. $k$ in Figure 3 (b). It can be seen from the plot that the $95 \%$ quantile for $\theta$ initially decreases marginally with $k$ but then stabilizes. According to the DIC criterion we chose $k=17$ as our best choice for the hyperparameter $k$. We present a plot of the posterior cummulative distribution function $(\mathrm{CDF})$ for this model in Figure 4.

Table 4: Posterior summary statistics for $\theta$ for model M3 with $\nu=6$ for different values of the hyperparameter $k$.

\begin{tabular}{r|r|r|r|r}
\hline \hline $\mathrm{k}$ & mean & sd & $95 \%$ ile & DIC \\
\hline 12 & 14.7 & 2.2 & 18.3 & 4762.8 \\
17 & 14.7 & 2.1 & 18.2 & 4753.4 \\
25 & 14.8 & 2.0 & 18.1 & 4784.5 \\
50 & 15.0 & 1.9 & 18.0 & 8252.0 \\
\hline \hline
\end{tabular}

Based on the multivariate $t$ model with $\nu=6$ and $k=17$ as selected using the DIC criterion above, we also assessed the sensitivity of the results to the prior specifications on the population means vectors. To 
(a)

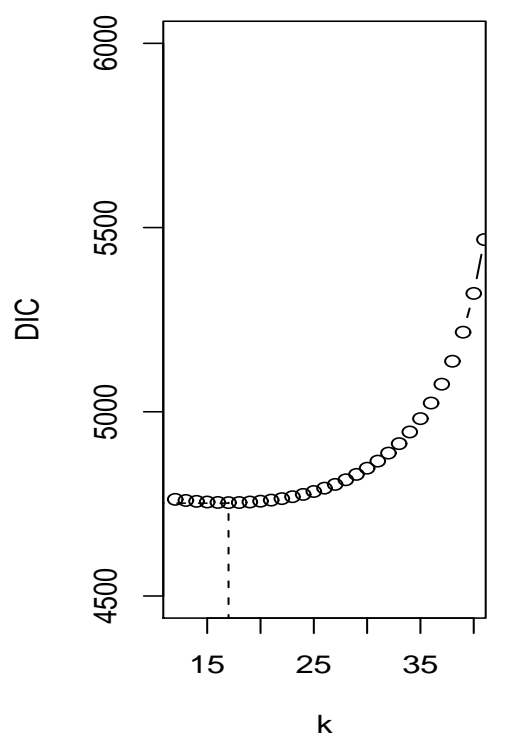

(b)

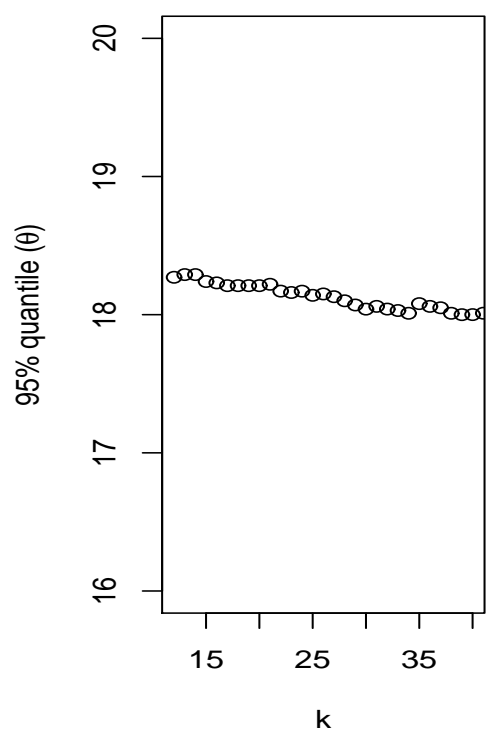

Figure 3: For model M3*, panel (a) presents DIC vs. $k$ and panel (b) presents the $95 \%$ posterior quantile of $\theta$ vs. $k$, where $k$ is the prior parameter defined in (3.3)

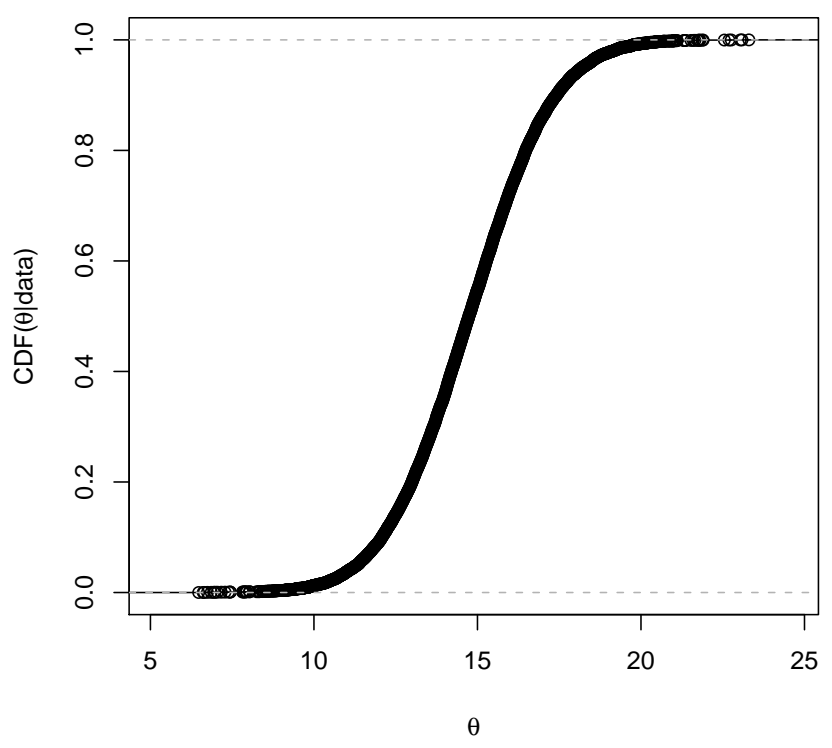

Figure 4: Plot of posterior CDF for $\theta$ for model M3* with $k=17$ 
do this, we analyzed the data for several plausible combinations of the prior mean vectors $\boldsymbol{\mu}_{1}$ and $\boldsymbol{\mu}_{2}$. Table 5 summarizes these results. It can be seen that the results for neutral, optimistic, and skeptic priors do not differ significantly. The posterior means are slightly different but the DIC and the statistic of interest, posterior $95 \%$ quantile for $\theta$, are close to each other. Thus, our methodology appears to be reasonably robust to the specification of the prior means.

These results together show that our $t$ model as selected above using the DIC criterion is fairly robust to the distributional assumptions and prior specifications.

Table 5: Posterior summary statistics for $\theta$ (model M3) with $\nu=6$ and $k=17$ for different prior specifications on population means.

\begin{tabular}{r|l|l|r|r|r|r|}
\hline \hline Prior & $\mu_{10}$ & $\mu_{20}$ & mean & sd & $95 \%$ ile & DIC \\
\hline Neutral & $(0,0,0,0,0,0,0,0,0,0)$ & $(0,0,0,0,0,0,0,0,0,0,0)$ & 14.7 & 2.1 & 18.3 & 4753.8 \\
Optimistic & $(-1,-1,-1,-1,-1,-1,-1,-1,-1,-1)$ & $(0,0,0,0,0,0,0,0,0,0,0)$ & 14.5 & 2.1 & 18.0 & 4754.2 \\
& $(-2,-2,-2,-2,-2,-2,-2,-2,-2,-2)$ & $(0,0,0,0,0,0,0,0,0,0,0)$ & 14.3 & 2.1 & 18.0 & 4754.6 \\
Skeptic & $(1,1,1,1,1,1,1,1,1,1)$ & $(0,0,0,0,0,0,0,0,0,0,0)$ & 15.0 & 2.1 & 18.4 & 4753.4 \\
& $(2,2,2,2,2,2,2,2,2,2)$ & $(0,0,0,0,0,0,0,0,0,0,0)$ & 15.2 & 2.1 & 18.6 & 4753.0 \\
\hline \hline
\end{tabular}

\section{Conclusion and Discussion}

The standard way of analyzing QT data involves comparing the $90 \%$ two-sided confidence intervals for the time-matched mean differences to the regulatory threshold of $10 \mathrm{~ms}$ at each time-point, and is overly conservative. Anand and Ghosh (2008) proposed a Bayesian approach to solving this problem by constructing an interval estimate for the maximum of the time-matched mean differences $\theta$ based on the derived samples from its posterior density. This method, along with few other recently proposed methods, assumes multivariate normality with a common covariance structure, which may be violated with small sample sizes. We have proposed a flexible class of multivariate $t$ models that includes the ubiquitous multivariate normal model for such data. We have compared these models using the DIC criterion that evaluates a model on the basis of its posterior predictive error.

We have gone in a methodical way to select the best model and then we show the robustness of our models and analysis methods to changes in distributional assumptions and prior specifications. For 
the given dataset, the DIC driven selection yields a small estimate for degrees of freedom $(\nu=6)$ which indicates that a multivariate normal model may not be a good fit for the data. A large estimate for $\nu$ would make the multivariate normality assumption more reliable. This methodology also gives us a way to check the applicability of the assumption of a common covariance structure for drug and placebo. A DIC-based criterion for the selection of the hyperparameter $k$ helps in discarding models with poor predictive abilities. The flexibility to run the analysis for different specifications for the prior mean vectors $\mu_{1}$ and $\mu_{2}$ enables one to guard against the worst case scenario with a skeptic prior when the historical data suggest that the drug prolongs QTc.

There does not seem to be an easy way of analyzing the QT data using a frequentist approach when the normality assumption of the likelihood is questionable. Even when the multivariate normal likelihood assumption is reasonable, the analysis using frequentist methods is not straightforward when the covariance structures for drug and placebo are different. We have provided a flexible class of models for the QT data and a sound mechanism for model building and analysis for such a data using a Bayesian approach. With the MCMC sampling capabilities of Wingbugs the analysis is easy to perform with standard software without relying on any kind of approximations or large sample theory assumptions.

\section{References}

Anand, S. P. and Ghosh, S. K. (2008). A Bayesian methodology for investigating the risk of QT prolongation, Journal of Statistical Theory and Practice, (In review).

Boos, D., Hoffman, D., Kringle, R., Zhang, J. (2007). New confidence bounds for QT studies, Statist. Med., 26, 3801-3817.

Box, G. E. P. (1949). A General Distribution Theory for a Class of Likelihood Criteria, Biometrika 36, 317-346.

Casella, G. and Berger, R. L. (2002). Statistical inference, Duxbury Press.

Eaton, M. L., Muirhead, R. J., Mancuso, J. Y., Lolluri, S. (2006). A confidence interval for the maximal mean QT interval change due drug effect, Drug Inf J., 40, 267-271. 
International Conference on Harmonisation (2005). Guidance for Industry: E14 Clinical Evaluation of QT/QTc Interval Prolongation and Proarrythmic Potential for Non-Antiarrythmic Drugs.

Available at: www.fda.gov/cber/gdlns/iche14qtc.htm

Patterson, S. (2005). Investigating Drug-Induced QT and QTc Prolongation in the Clinic: A Review of Statistical Design and Analysis Considerations: Report from the Pharmaceutical Research and Manufacturers of America QT Statistics Expert Team, Drug Inf J., 9, 243-266.

Patterson, S., Jones, B. Zariffa, N. (2005). Modeling and interpreting QTc prolongation in clinical pharmacology studies, Drug Inf J., 39, 437-445.

Patterson S. and Jones B. (2005) Bioequivalence and Statistics in Clinical Pharmacology, Chapman and Hall, CRC Press; London.

Spiegelhalter, D. J., Best, N. G., Carlin, B. P., and Van der Linde, A.(2002). Bayesian Measures of Model Complexity and Fit (with Discussion), Journal of the Royal Statistical Society, Series B, 64(4), 583616. 